$\mathbb{T}$ periodica polytechnica

Transportation Engineering

$4 1 / 1 ( 2 0 1 3 ) \longdiv { 3 9 } 4 3$

doi: 10.3311/PPtr.7095

http://periodicapolytechnica.org/tr

Creative Commons Attribution (1)

RESEARCH ARTICLE

\section{Experimental verification of vehicle platoon control algorithms}

\author{
Szilárd Aradi / Gábor Rödönyi / Péter Gáspár / Zoltán Hankovszki / Roland Kovács
}

Received 2012-10-27

\begin{abstract}
Organizing a group of vehicles into a vehicle platoon in a way that, except for the leading vehicle, each platoon member can be autonomously driven has been a research goal for decades. Among other benefits this results in a decrease of fuel consumption and also in the driver's workload and an increase in a better use of road capacity. The recent developments in the area of active control systems for vehicles make it possible to realize more and more autonomous functions and the above defined cooperation between vehicles seems to be increasingly feasible. This article aims to point out that today it is possible to reach this goal without vehicle specific software and hardware.
\end{abstract}

\section{Keywords}

vehicle platoon $\cdot$ string stability

\section{Acknowledgement}

This work is supported by the Hungarian National Office for Research and Technology through grants TECH_08_2/2-20080088, OM-0239/2008, which is gratefully acknowledged.

\section{Szilárd Aradi}

Department of Control for Transportation and Vehicle Systems, Budapest University of Technology and Economics, Stoczek u. 2, H-1111 Budapest, Hungary

e-mail: aradi.szilard@mail.bme.hu

\section{Gábor Rödönyi}

\section{Péter Gáspár}

Systems and Control Laboratory, Computer and Automation Research Institute, Hungarian Academy of Sciences, Kende u. 13-17, H-1111 Budapest, Hungary

\section{Zoltán Hankovszki}

Roland Kovács

Knorr-Bremse R\&D Center, Major u. 69, H-1119 Budapest, Hungary

\section{Introduction}

It is widely proposed to group vehicles in platoons in such a manner that each vehicle except the lead can be autonomously controlled. The control aim could be a decrease in fuel consumption, the driver's workload or an increase in road capacity. The main projects of this active research field originating from the '80s are the Californian PATH program,the MOC-ITS in Japan where intelligent highway systems and road infrastructure were developed to improve traffic safety and enhance transportation efficiency. The European project SARTRE was based on the existing road network and was oriented towards the development of the vehicles.

In the Hungarian TruckDAS project an automated vehicle platoon of heavy vehicles was developed. The goal of the project was to analyze the control algorithms and synthesize the experimental results, see [2]. Within the framework of the present project we are developing a system specifically designed for commercial vehicle platforms, where traditional positioning methods (e.g. GPS) are managed together with other data (e.g. speed, lateral acceleration, radar) supplied by sensors on the vehicle. An important purpose of the project is to achieve the desired decrease in the number of accidents even with strict economy requirements and demanding circumstances through the new driver assistance functions. In our experience in a platoon of three vehicles a safety gap of $8 \mathrm{~m}$ proved to be safe under the following conditions: The deceleration of the leader vehicle is not greater than $2 \mathrm{~m} / \mathrm{s}^{2}$ and there is a dwell time between intensive acceleration and abrupt braking so that transients can cease.

It should be mentioned at this point that more accurate solutions of platoon controller exist (see [4-6]), but in all cited cases a sufficiently precise and detailed knowledge about the model of the drive-line and the brake system is required, and beyond that, the direct control of the brake cylinder pressures and throttle valve of the engine. These models are hard to gain, they depend on changing technical conditions of the aging vehicle. Furthermore, the direct control of actuators, if possible at all, would interfere with the existing commercial control units. In contrast to the former solutions, the explicit goal of the research 
was to use only standardized and general functions available on every modern commercial heavy truck. Such services are the execution of external deceleration demands provided by EBS, and external engine torque demands provided by ECU. Utilizing existing, standardized services allows the widespread applicability of the platooning concept.

The remainder of the paper is organized as follows. In Section 2 the experimental environment and the platoon architecture are presented. In Section 3 the components of the control system are shown. In Sections 4 the principle of the control design is detailed. In Section 5 the steps of control design are briefly sketched. In Section 6 the failure modes and their handling are analyzed. In Section 7 some conclusions are provided.

\section{Experimental Environment}

The designed controllers are implemented on a platoon of three heavy trucks and tested on a 3-km-long runway. The leader vehicle, driven by a driver, is a MAN TGA two-axle tractor of 18 ton with a load cage. The second vehicle is a Volvo FH 24 ton three-axle truck. The third one is a Renault Magnum two-axle tractor of 18 ton with a semitrailer, see Fig. 1. It is important to note that all vehicles are equipped with automatic gear change, thus acceleration can be attained purely by software.

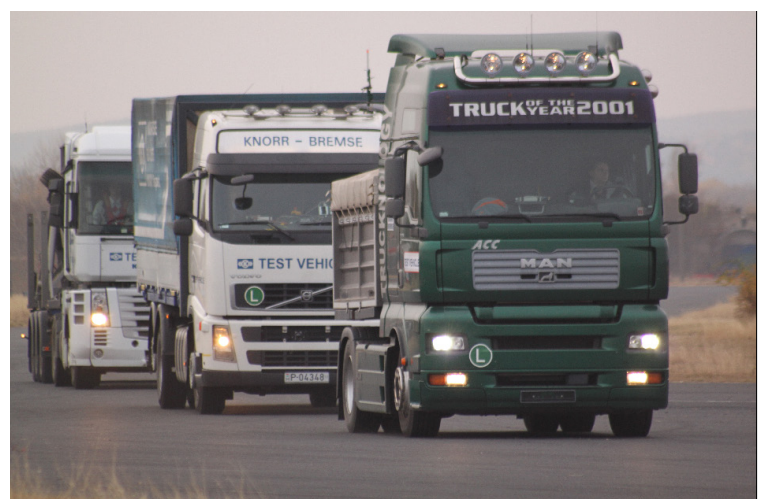

Fig. 1. Experimental environment: MAN TGA, Volvo FH, Renault Magnum

\subsection{Communication network}

The communication network specified in this section is applied only for experimental purposes because of its ideal properties and ease of handling. It consists of radio transceivers operating on the open $868 \mathrm{MHz}$ ISM narrow-band. On the physical layer of the network model GFSK modulation and Manchester coding are used, while the data link layer is based on 4 byte device addressing and CRC-based data protection. In the network and data layers broadcast messaging is applied which means that there is no peer-to-peer socket communication and classic network topology. With proper timing strategy, all peers receive all messages of the other network nodes. The channel access is achieved by time division multiple access (TDMA): The leading vehicle initiates the broadcast, and the two followers accept it. As soon as the channel turns disengaged after the end of this broadcast, the second vehicle sends its message and so the cycle is restarted. In the top network layer, compact data description is utilized where the data is stored in the form similar to the representation of the CAN network protocol. Thus, the data packet is only 30 bytes long, which is extended with a packet identifier for further data-protection purposes.

\subsection{Platoon architecture}

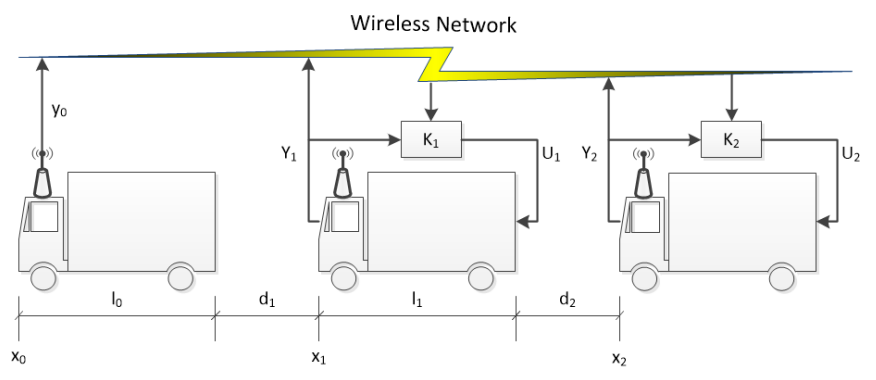

Fig. 2. Control structure of the vehicle platoon

The control scheme of the platoon is plotted in Fig. 2 The lead vehicle is driven by a driver. In the other, follower vehicles acceleration and braking tasks are carried out by on-board controllers based on information coming from a radar and a communication network. The radar provides relative speed and distance with respect to the preceding vehicle. Through the communication network,each vehicle transmits longitudinal acceleration and speed to its follower vehicle and the leader provides pedal signals, speed and filtered GPS position for all. Communication allows to quicken the reaction to maneuver changes. The controllers, denoted by $K_{i}$, calculate acceleration reference $u_{i}$ which is transmitted after checking its domain and rate to the EBS as deceleration demand, on the one hand, and to the engine control system in the form of engine torque demand, on the other hand.

\section{Model of the Longitudinal Dynamics}

The acceleration of a vehicle can be described by equation $a=\frac{1}{m}\left[F_{a}+F_{g}+\frac{1}{h}\left(M_{r r}+\tau_{b}+\tau_{e}\right)\right]$, where $m$ denotes vehicle mass, $M_{r r}$ is the rolling resistance, $F_{a}$ and $F_{g}$ are the air drag and gravitational force due to road inclination, $\tau_{e}$ and $\tau_{b}$ stand for driving and, braking torques and $h$ for effective wheel radius.

\subsection{Powertrain}

The longitudinal behaviour of the drive-line is highly nonlinear and hybrid dynamics due to the engine speed dependent torque characteristics, gear dependent torque transmission and the gear change process. Additionally, the control systems of both the automatic gear change and the engine function as finite state machine. Intervention possibility for the platoon control software is provided by a torque demand addressed to the engine control unit through CAN interface. 


\subsection{Brake system}

The dynamics between the prescribed brake pressure as input and the brake force as output can be described by an input delay necessary for building up the brake cylinder pressures, and a linear time-invariant system. For a given deceleration reference, the computation of the necessary brake pressure for each individual wheel requires knowledge of the vehicle mass, load distribution, resistances, wheel radius, incline angle of the road and tire-road adhesion characteristics. We can assume that all the necessary estimation problems are performed by the EBS, which can receive a deceleration demand that is carried out by a finite state machine.

\subsection{Model of the longitudinal dynamics}

As shown above, both the drive-line and the brake system are complex non-linear and hybrid systems with mostly unknown details, but they can be approximated with the following LTI system

$$
P_{i}(s)=e^{T_{d} s} \frac{k_{i} s+1}{s^{2}+2 \xi_{i} \omega_{i 0} s+\omega_{i o}^{2}} c_{i}^{d / b}
$$

where the $T_{d}$ is the delay, $k_{i}, \xi_{i}, \omega_{i 0}$ and $c_{i}^{d / b}$ are identifiable parameters $\left(c_{i}^{d / b}=c_{i}^{d}\right.$ by driving and $c_{i}^{d / b}=c_{i}^{d}$ by braking $)$.

\section{Control of Vehicle Platoon}

At the upper level (platoon) a global algorithm must ensure the string stability of the platoon. At the bottom level (vehicle) the required control tasks must be guaranteed. String stability means that spacing errors due to non-zero initial conditions are not amplified along the platoon and in time.

\subsection{String stability}

Assume that the longitudinal dynamics of the vehicle platoon is characterized by an LTI system. The spacing error of the $i^{\text {th }}$ vehicle is denoted by $e_{i}$ (where $\mathrm{i}=0$ is the leader vehicle), $H_{i}(s)=\frac{e_{i}(s)}{e_{i-1}(s)}$ is the transfer function of the spacing error propagation and the $h_{i}(t)$ is its impulse response. In the case of three vehicles (i=2) the vehicle string is stable, if $\left\|H_{i}(s)\right\|_{\infty} \leq 1$. In this case $\left\|h_{i}(t)\right\|_{1} \leq 1$, i.e., the amplitude of the spacing error can not increase $\left(\left\|e_{i}\right\|_{\infty} \leq\left\|e_{i-1}\right\|_{\infty}\right)$.

\subsection{Control algorithm}

Assume that vehicle dynamics is ideal

$$
\dot{x}_{i}=v_{i}, \quad \dot{v}_{i}=a_{i}, \quad a_{i}=u_{i}
$$

where $x_{i}, v_{i}, a_{i}$ and $u_{i}$ denote the position, velocity, acceleration and acceleration demand of the controller of the $i^{\text {th }}$ follower vehicle. Let $L_{i}$ denote the desired inter-vehicular spacing of $j^{t h}$ vehicle and define the following sliding surface

$$
S_{i}:=\dot{e}_{i}+q_{i} e_{i}+q_{3}\left(v_{i}-v_{0}\right)+q_{4}\left(x_{i}-x_{0}+\sum_{j=1}^{i} L_{j}\right)
$$

with parameters $q_{1}, q_{3}, q_{4}$. If $S_{i} \rightarrow 0$, then $e_{i} \rightarrow 0$. The controller is chosen to converge $S_{i}$ to zero. Choose a parameter $\lambda>0$ and from the condition $\dot{S}_{i}+\lambda S_{i}=0$ the required control input $u_{i}$ can be computed.

The system is formed adaptively on the changes of the air and rolling resistances if the control input is modified according to the following form

$$
u_{i}:=\hat{M}_{r r, i}+h \hat{c}_{a, i} \hat{v}_{i}^{2}+\hat{m}_{i} u_{i}
$$

where $\hat{M}_{r r, i}, \hat{c}_{a, i}$ (drag coefficient) and $\hat{m}_{i}$ (vehicle mass) parameters are detailed in [1].

\section{Control Design for Vehicle Platoon based on Mea- surements}

In order to enable that any vehicle to join the platoon, the controller must be independent of the specific parameters and the actual loads of the vehicles. The parameters of the controller are determined on the basis of the nominal vehicle model. On one hand the differences must be handled individually on every vehicle (parameter estimation and engine characteristic), on the other hand the controller must be robust against the differences between the vehicles.

\subsection{Design at the vehicle level}

The dynamics of braking, and even more the characteristics of the powertrain are significantly different from the identified model. In the former case the model error is caused by the nonlinear and hybrid behaviour of the EBS software. In the case of the powertrain the non-linear characteristics of the engine and the software of the powertrain show differences. The controller must be robust against the software errors. The non-linearity of the engine can be compensated by having the $u_{i} \rightarrow a_{i}$ transfer in steady-state as

$$
u_{i}:=c u_{i}
$$

where $\mathrm{c}$ is a possibly gear dependent constant. In that way the acceleration demand could be adapted according to the characteristics of the engine.

A much more complicated problem is caused by the gear shifting process. For example assume that the leader vehicle is accelerating. During the gear shift its measured acceleration $\left(a_{0}\right)$ is terminated moreover its sign will be negative because of the resistances. As a result the follower vehicles cease accelerating. After the gear shift the leader will accelerate onward. The follower vehicle, which have to shift then, will accelerate stronger to decrease the spacing error. This behaviour can be eliminated if we use $u_{i-1}$ instead of $a_{i-1}$ and instead of $a_{0}$ we use the acceleration demand from the pedal signal of the leader vehicle. These signals cannot be eliminated during the gear shifting process, therefore each vehicle can shift in its own way.

\subsection{Tuning of control parameters}

After the identification of the simplified model the parameters $q_{1}, q_{3}, q_{4}$ and $\lambda$ can be calculated on the basis of the simulations and numerical analysis. A possible method could be choosing an objective function by running simulations with specific 
maneuvers, in which the spacing errors and the control signals are taken into account whilst the string stability and the maximum of the control signals could be specified as constraints. In the parameters the non-linear objective functions are minimized with constraints. The drawback of the method is the necessity of the tuning of the parameters during the real tests to which this method can not provide a solution.

For this reason we divide the problem into two tuning tasks each having only two parameters. When the platoon is string stable, the spacing error of the first follower has the largest peak. The controller depends on two constants

$$
k_{1}:=\left(q_{1}+q_{4}+\lambda+\lambda q_{3}\right) /\left(1+q_{3}\right) ; \quad k_{2}:=\lambda\left(q_{1}+q_{4}\right) .
$$

In the first task the dynamics of the $a_{0} \rightarrow e_{1}$, the damping, the maximum spacing error and the maximum control signal can be determined. In the second task we examine the effect of the $q_{1}$ and $q_{4}$ on the string stability, i.e., the impulse response of the $H_{i}(s)$.

\section{Failure modes and their handling}

As mentioned above the goal of the project was to analyse the control algorithms and synthesize the experimental results. So far we have assumed the faultless operation of the system components and normal environment conditions. We have analysed and experimentally tested the control strategy operating in normal mode, and two other strategies in faulty network modes, see Fig. 3. Upper bounds on the worst case spacing errors have also been computed for platoons of heterogeneous dynamics, which can support the selection of the safety gaps, see [8,9].

The effects of failures and special conditions have not been analysed or tested, furthermore the communication network specified and implemented in the project was intended for demonstration purposes only.

First we must analyse the abnormal operations and their effects. In former research [10 12] the abnormal operating conditions were divided into two main groups:

1 Hard Faults: these include failures or faults in one of the control system components, such as mechanical failures in the vehicles, failures in sensing, communication, control and actuation.

2 Soft Faults: these include non-ideal environmental conditions, such as rain, fog, snow, etc. and the loss of performance due to the wear of the system components.

According to these faults the control system must switch between two main operating mode classes: normal mode, which guarantees optimal performance under nominal conditions, and abnormal mode, which contains several degraded modes. The latter modes must be capable of ensuring safety and minimizing performance degradation.

In modern commercial vehicles the on-board sensors can detect and diagnose their own failures. Furthermore the result of
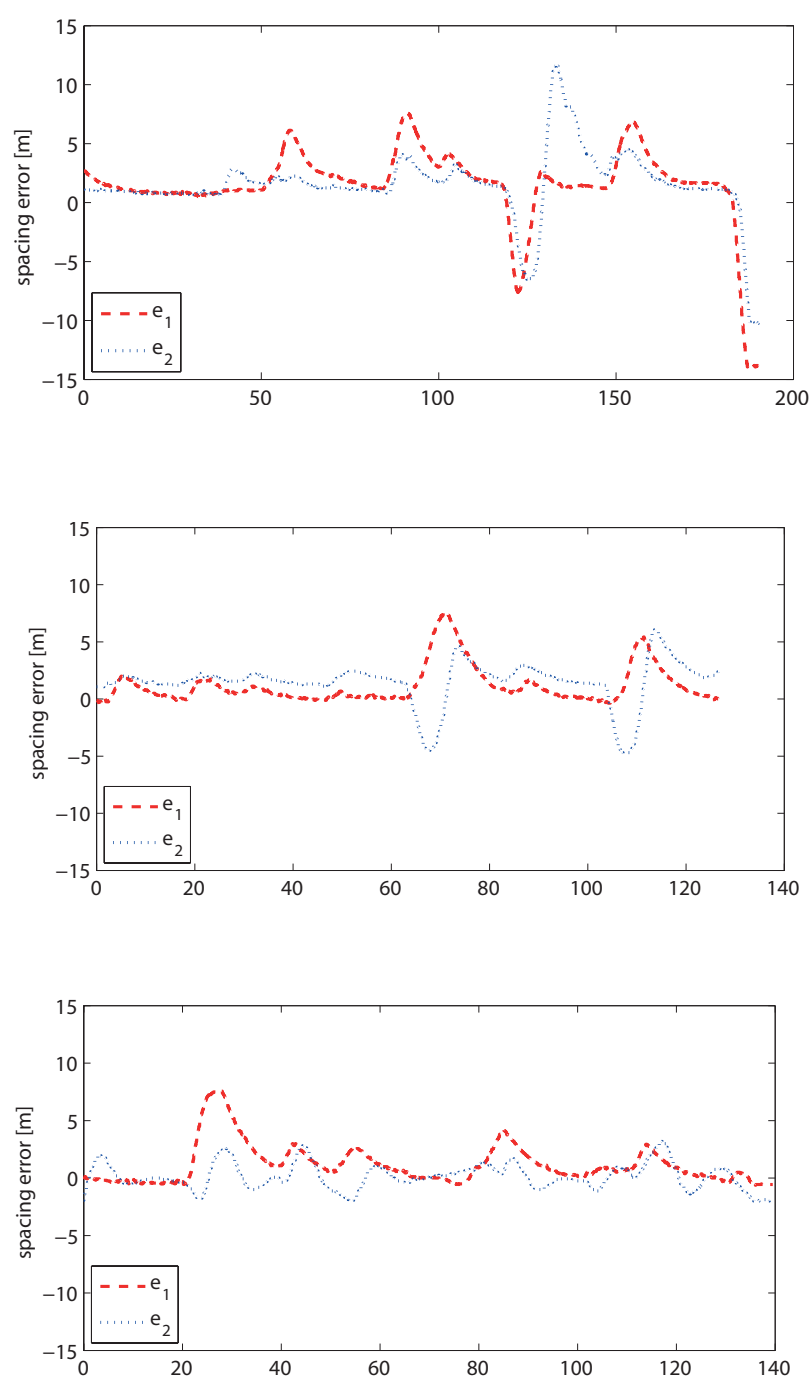

Fig. 3. Spacing errors

this self-diagnostic is also available on the CAN bus similarly to the normal sensor signals. These sensor failures could be the following:

- Radar sensor fault: When the radar sensor has developed a fault, it is possible to use the velocity and the GPS position received via the communication channel to estimate the relative velocity and distance to the vehicle ahead.

- Wheel speed sensor fault: In the case of an Electronic Brake System (EBS) it results in a special scenario because of the changed operating mode of the brake system. The EBS switches to a degraded mode, while the ABS and the ESP functionalities are not available. From the point of view of platooning the effect of the brake performance loss must be examined accurately.

- GPS fault: If the GPS receiver is faulty, the position can be calculated from the combination of the radar sensor measurements and the communication information.

The other failure class contains the network faults. As we have mentioned above we used an ISM narrow-band radio in 
PHY/MAC, and broadcast messaging in the network and data layers without peer-to-peer socket communication and classic network topology. The channel access is achieved by a tokenlike protocol. These network could be formulated in a simple way. We only note that it has a sampling time of $T=N T_{s}$, $N=10$, and the packet transmission takes about $d=h T_{s}$. In certain conditions, no packet loss occurs and as the transmission delay $d$ has small variation, it can be considered as constant. If $y(k)$ denotes the variable to be transmitted at the network input, then

$$
\hat{y}(k)= \begin{cases}y(k-h) & \text { if } \frac{k-h}{N} \text { is an integer } \\ \hat{y}(k-1) & \text { otherwise }\end{cases}
$$

denotes the network output at the receiver.

Nevertheless for commercial purposes special communication devices must be used according to the ITS standards, for example Wireless Access in Vehicular Environments/Dedicated Shortrange Communication (WAVE/DSRC) standards. They are composed of IEEE802.11p for PHY and MAC layers and IEEE 1609-family standards to cover additional layers in the protocol suite. In that case and in a noisy environment the network model is more complex with several uncertainties such as variable transmission time, packet losses and other network errors.

The network and sensor faults must be detected and handled by switching between different control strategies. The faults must be defined accurately, according to their timeliness, i.e., if it is a temporary or a permanent fault (eg. radar signal loss in curves). The formulation and implementation of a refined network model and emergency switching protocols are needed. The failure effect must be examined by simulation with special regard to the transients and worst case scenarios.

\section{Conclusions}

It has been demonstrated that the currently available on-board services of commercial heavy trucks can be applied to create new platooning services. Without detailed vehicle models, output-feedback controllers that provide satisfactory tracking performance for heterogeneous platoons can be designed. The feed-through of control inputs, speeds and filtered GPS positions have the benefit of reducing the safety gaps between the vehicles. Degraded controller modes corresponding to network channel faults have been also analysed and verified in experiments. Nevertheless in a commercial system traffic safety is the most important consideration. In order to handle the network faults a more realistic network model must be developed and the extended system must analysed.

\section{References}

1 Swaroop D, Hedrick JK, String stability of interconnected systems, IEEE Transactions on Automatic Control, 41(3), (1996), 349-357, DOI 10.1109/9.486636

2 Project TruckDAS/Platooning, Innovation of distributed driver assistance systems for commercial vehicle platform, November, 2011, http://wWW. truckdas.hu/

3 Swaroop D V A H G, String Stability of Interconnected Systems: An Application to Platooning in Automated Highway Systems, $\mathrm{PhD}$ thesis, University of California at Berkeley, (1994).

4 Nouveliere L, Mammar S, Experimental vehicle longitudinal control using a second order sliding mode technique, Control Engineering Practice, 15(8), (2007), 943-954, DOI 10.1016/j.conengprac.2006.11.011

5 Gerdes JC, Hedrick JK, Vehicle speed and spacing control via coordinated throttle and brake actuation, Control Engineering Practice, 5(11), (1997), 1607-1614, DOI 10.1016/S0967-0661(97)10016-8

6 Rajamani R, Vehicle dynamics and control, Springer, Mechanical Engineering Series, 2006.

7 Németh B, Gáspár P, Lpv-based control design of vehicle platoon considering road inclinations, Proc. of the 18th IFAC World Congress, Milan, Italy, (2011).

8 Rödönyi G, Gáspár P, Bokor J, Aradi S, Hankovszki Z, Kovacs R, Palkovics L, Analysis and experimental verification of faulty network modes in an autonomous vehicle string, 20th Mediterranean Conference on Control and Automation (MED), Barcelona, Spain, (2012), 884-889, DOI 10.1109/MED.2012.6265750

9 Rödönyi G, Gáspár P, Aradi S, Hankovszki Z, Kovacs R, Palkovics L, Guaranteed peaks of spacing errors in an experimental vehicle string, Proc. of the 7th IFAC Symposium on Robust Control Design, Aalborg, Denmark, (2012), 759-764.

10 Godbole D, Lygeros J, Singh E, Deshpande A, Lindsey A, Communication protocols for a fault-tolerant automated highway system, IEEE Transactions on Control Systems Technology, 8(5), (2000), 787-800, DOI 10.1109/87.865852

11 Lygeros J, Godbole D, Broucke M, A fault tolerant control architecture for automated highway systems, IEEE Transactions on Control Systems Technology, 8(2), (2000), 205-219, DOI 10.1109/87.826792

12 Yi J, Howell A, Horowitz R, Hedrick K, Alvares L, A Fault Tolerant Control Architecture for Automated Highway Systems, California PATH Research Report, (2001). 